\title{
Research on Power Demand Forecasting Methods
}

\author{
Zhigang Zhan \\ Electric Engineering North China Electric Power University, Baoding 071003, China. \\ 3469845175@qq.com
}

\begin{abstract}
In recent years, with the rapid economic development of developing countries, the contradiction between economic development and resources and environment has become increasingly acute. It is of great practical significance to study the power market demand forecasting method for energy conservation and emission reduction. First of all, this paper draws on the technology and management experience of developed countries in energy conservation, energy conservation and emission reduction, and combines the actual economic development faced by developing countries to make predictions according to the classification of end users. In addition, taking into account the application of energy-saving and clean energy, we should grasp the changing trend of electricity consumption, make better power distribution and make contributions to energy conservation and environmental protection. Secondly, the correlation analysis and time series forecasting model are used to predict the demand of electricity in the region. In addition, this paper selects a representative country from both developing and developed countries to compare power demand consumers and analyze power demand in later period. Finally, this paper conducts sensitivity analysis on the power demand model based on correlation analysis and time series prediction, and obtains the applicability of the model. In the future, the forecasting methods of the models will be optimized to contribute to the global energy utilization and power energy prediction.
\end{abstract}

Keywords: Time series prediction, correlation analysis, power demand forecasting.

\section{Introduction}

Electric power forecasting is the use of historical data to predict future time of power consumption, energy management system (EMS) is an important part of power system in the developing countries and developed countries has become indispensable to modern management factors in the management, in power system for regional power transmission scheme and load scheduling plays an important role. The application of forecasting model can effectively improve the accuracy of power forecasting and make the power system run economically.

\section{Model Establishment}

\subsection{Correlation Analysis}

In order to predict the growth of power demand, we selected the main factors related to the growth of power demand, and carried out correlation analysis to optimize the model

Table 1. Degree of Correlation Tables

Relevance

\begin{tabular}{cl}
\hline$|\rho|=0$ & Completely unrelated \\
$0<|\rho|<0.3$ & Weak correlation \\
$0.3<|\rho|<0.5$ & Low correlation \\
$0.5<|\rho|<0.8$ & Significant correlation \\
$0.8<|\rho|<1$ & Highly correlated \\
$|\rho|=1$ & Fully correlated \\
\hline
\end{tabular}


The correlation coefficient is Precipitation, temperature, population, government factors, and other related indicators correlated with the Prediction of the moment of the critical point four degree of importance, as shown in Table7.

Table 2. Relevance List

\begin{tabular}{lcccc}
\hline & Prediction & Temperature & population & Energy technology development \\
\hline & & & & \\
\hline $\begin{array}{l}\text { The correlation } \\
\text { coefficient }\end{array}$ & 8.263 & 1.122 & 3.789 & 3.223 \\
Relevance & Highly correlated & Low correlated & Highly correlation & Highly correlation \\
\hline
\end{tabular}

It can be seen from the above table that precipitation and temperature are the most important factors in predicting the critical point in the climate. Here we will conduct a time series prediction to predict the demand of electricity in the region

\subsection{Time Series Prediction}

Time series prediction technology is to study the change trend of the predicted target through the processing of its own time series. A time series is usually the superposition or coupling of the following types of changes.

Let the observation sequence be $y_{1}, \ldots y_{t}$, take the number of moving averages $\mathrm{N}<\mathrm{T}$, the calculation formula of a simple moving average is

$$
\begin{aligned}
M_{t} & =\frac{1}{N}\left(y_{t}+y_{t-1}+\cdots y_{t-N+1}\right) \\
& =M_{t-1}+\frac{1}{N}\left(y_{t}-y_{t-N}\right)
\end{aligned}
$$

When the basic trend of the forecast target is up and down at a certain level, a simple moving average method can be used to build the prediction model

$$
\begin{gathered}
y_{t+1}=M_{t}=\frac{1}{N}\left(y_{t}+\cdots+y_{t-N+1}\right), t=N, N+1, \cdots, T, \\
S=\sqrt{\frac{\sum_{t=N+1}^{T}\left(y_{t+1}-y_{t}\right)^{2}}{T-N}}
\end{gathered}
$$

Set the time series as $y_{1}, y_{2} \ldots y_{n}$; The weighted moving average formula is

$$
M_{t w}=\frac{w_{1} y_{t}+w_{2} y_{2}+\ldots+w_{N} y_{t-N+1}}{w_{1}+w_{2}+\ldots+w_{N}}, t \geqslant N
$$




\section{Regression Equation and Test}

Therefore, fitting the prediction and temperature datas and use MATLAB to plot the relationship

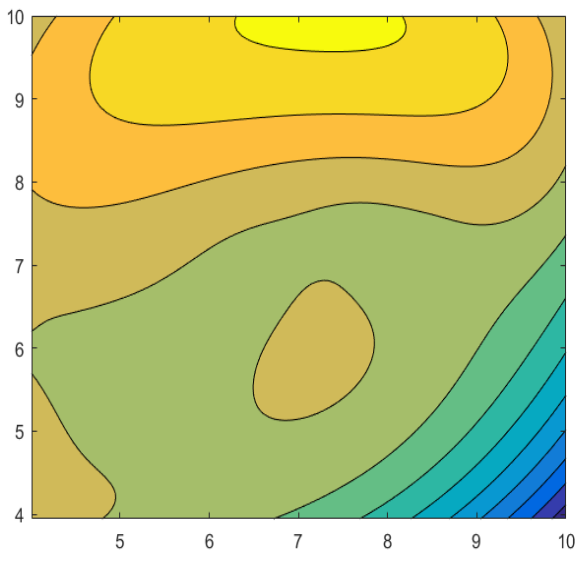

Fig. 1 Surface Top View

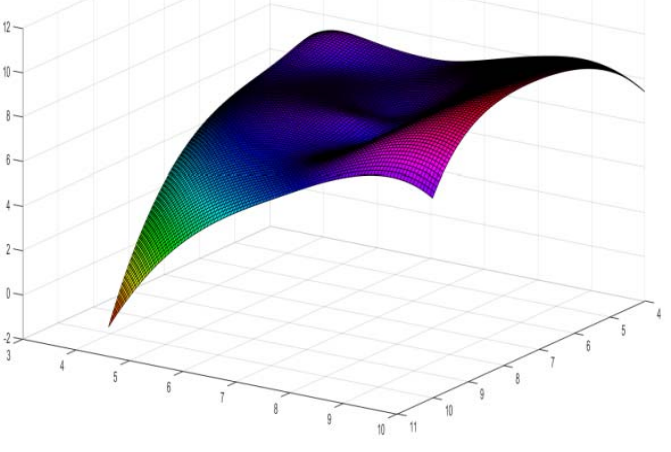

Fig. 2 3D Prediction Model Display

Finally, the correlation analysis and time series forecasting model are used to predict the demand of electricity in the region. Specific predictions are shown in the table below

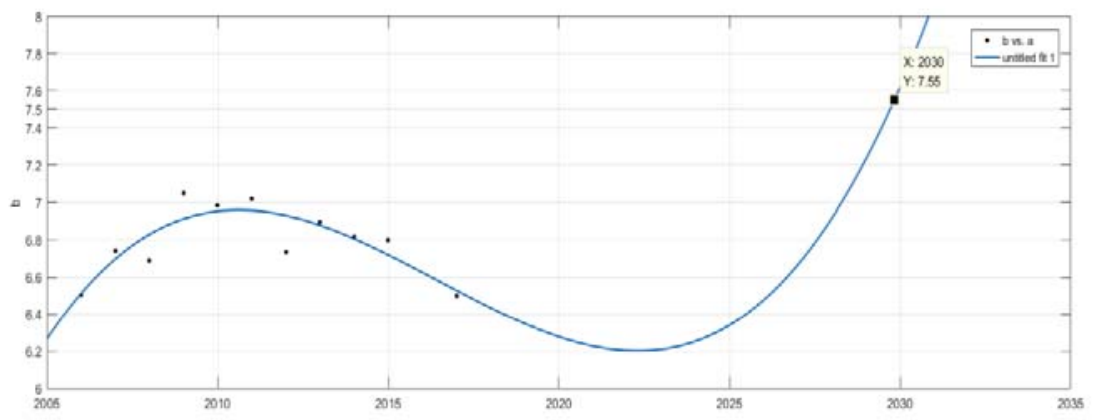

Fig.3 Specific Predictions are shown in the Table Below

\section{Test and analysis of models in large and small states}

We used the electricity demand forecasting model to predict the representatives of the developed and developing countries, collected Germany and China from 2007 to 2017, and predicted the next year. Using MATLAB to draw the power prediction curve and the actual difference, so that we can more directly predict.

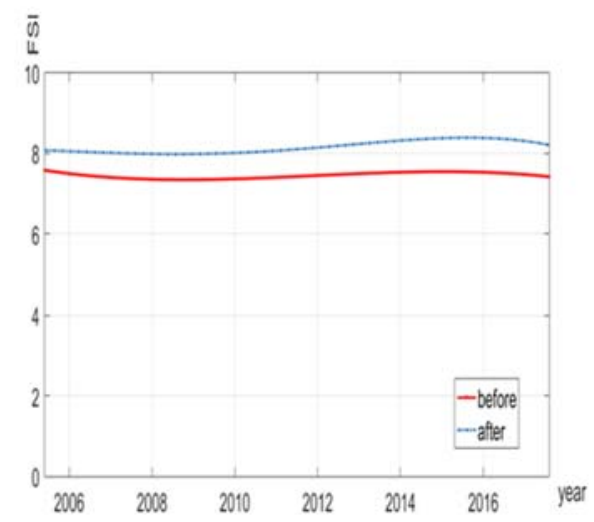

Fig.4 China's Forecast

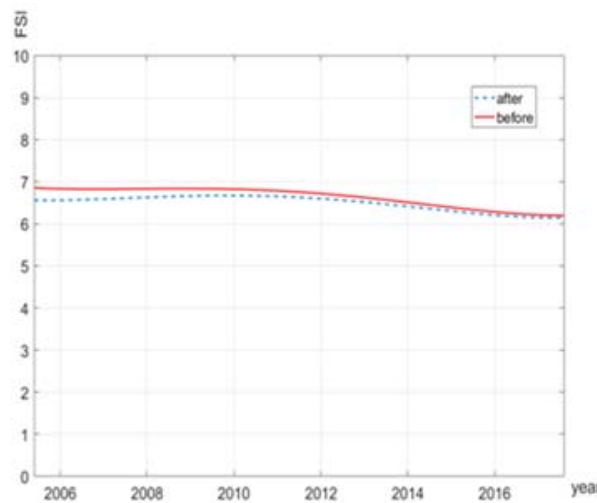

Fig.5 Germany's Forecast 


\section{Future Work}

1. Taking into account the impact of various new generation technologies in the mode

2. Considering more related factors that affect the power demand, the performance of the model is stronger.

3. Based on the existing energy prediction model, a more optimized distribution point market optimization model is proposed to contribute to the uneven distribution of power and energy conservation and emission reduction worldwide

\section{References}

[1]. Current status and future advances for wind speed and power forecasting By: Jung, Jaesung; Broadwater, Robert P. RENEWABLE \& Sustainable Energy Reviews Volume: 31 Pages: 762777 Published: MAR 2014.

[2]. Forecasting volatility of wind power production By: Shen, Zhiwei; Ritter, Matthias APPLIED ENERGY Volume: 176 Pages: 295-308 Published: AUG 152016.

[3]. Forecast combining with neural networks By: Donaldson, RG; Kamstra, M JOURNAL OF FORECASTING Volume: 15 Issue: 1 Pages: 49-61 Published: JAN 1996.

[4]. Non-Parametric Estimation of a Multivariate Probability Density by: Epanechn. Va Theory Of Probility And Its Applications, USSR Volume: 14 Issue: 1 Pages: 153-\& Published: 1969.

[5]. Electric Forecasting Using an Artificial Neural Network. Park D C et al. IEEE Transaction on PWRS. 1991.

[6]. Electric Load Forecasting Using an Artificial Neural Network. Park DC et al. IEEE Transactions on PWRS. 1991.

[7]. Integrating real and financial options in demand-side electricity contracts. ORON S S. Decision Support. 2001.

[8]. Artificial Intelligence in Short Term Electric Load Forecasting: a State-of-the-art Survey for the Researcher. Metaxiotis K, Kagiannas A, Askounis A, et al. Energy Conversion. 2003. 